\title{
Replacement of Human Labour With Integration of Machines Into a Self-Governing System
}

\author{
Kali Charan Rath, GIET University, India \\ Surya Narayan Maharana, GIET University, India \\ Jyoti Rajak, MSME Indo Danish Tool Room, India
}

\begin{abstract}
Advanced manufacturing runs with highly innovative technology and accommodates quality production without excessive capital investment. So, there are three main corners named. Efficient production, intelligent production, and effective organization are the special focus areas in today's advanced technology adopted by manufacturing sectors. The domain of an automated manufacturing system is not only limited to the production lines but also can be treated as a tool that automates plant inventory, orders, purchasing, or even marketing. Technological significance can be automated to improve the productions of the industry. The objective of this paper is to explore the concept of automation and presents brief taxonomies across multiple scientific and industrial automation systems. This article also describes the effect of automation on the society and environment.
\end{abstract}

\section{KEYWORDS}

AI, Application, Automation Management System, Automation, Automation System, Components of Automation, Lifestyle in Automation, MI

\section{INTRODUCTION}

Today's industrial processing systems, factories, machinery, test facilities, etc. turned from mechanization to automation thanks to the rapid climb in technology. A mechanization system desires human interference to work the manual operated machinery. Computerized automation control is being single-minded by the necessity for top accuracy, quality, precision, and performance of commercial processes due to the efficient control technologies evolved in running industrial revolution. Automation may be a technology handling the appliance of "Mechatronics" and "computer" for production of excellent services. Automation is generally classified into Manufacturing and service automation. Technology fuels country's economy, captures technological imaginations, and guarantees extraordinary prospects. It permeates most aspects of day-to-day activities (Faunce 1965). Rising technologies, together with AI, Machine Learning (ML), and advanced Artificial Intelligence (AI), have the potential to modify several tasks presently performed by researchers and industrial R\&D division (Balfe et. al. 2015; Dutta et. al. 2014; Norman 1978). The technology surroundings are concerned by implementing and interfacing varied data technologies (IT) and operational technologies (OT) (Heck et. al., 2003; Forge \& Blackman 2010; Sheridan 2011; Pan et. al. 2012).

The production value is a smaller amount that is helpful to the shoppers in an exceedingly approach that the value of the merchandise is a smaller amount. There is less demand of normal laborers, but 
a lot of demand of skilled persons for abundant easier and precise value product (Sjobakk et. al. 2014; Vagia et. al. 2016; Tarafdar et. al. 2017). 'Technological unemployment' is not new and has surfaced repeatedly since the economic revolution, as staff feared that new machines would possibly drive them out of jobs (Baumann et. al. 2018; Skorupski \& Uchronski 2018; Prettner 2019; Fallon et al. 2015). Automation may be a step on the far side the mechanization that makes use of high management capability devices for economical, producing or production processes (Lankisch et. al. 2019). The future role of the human operator with experienced knowledge and capabilities to assist the automation systems in support to Industry 4.0 is the focus area in recent technology (Muir 1987; Grossman et. al. 2017; Kizito et. al. 2020). Industrial automation is that the use of management systems, like computers or robots, and data technologies for handling completely different processes and machineries in a trade to interchange an individual's being. It is the second step on the far side mechanization within the scope of industrial enterprise.

While robotization helps money related blast, makes occupations, and improves staying guidelines, it can likewise introduce basic requesting circumstances for laborers and networks, which incorporates action relocation, interruptions to close by economies, changing over ability needs, and rising disparity (Abdollahi \& Ebrahimi 2019; Herrera et. al. 2019; Zelinka \& Amadei 2019; Li et. al. 2013; Baporikar 2020).

In relatively prepared and repetitive settings, duties most level of automation consist of bodily sports in addition to records collection and processing (Parasuraman et. al. 2008). This will surely consist of rethinking education and training, promoting jobs and protection nets, in addition to facilitating relocation for those dislocated. Individuals within the body of workers will need to speak more absolutely with computer systems as a part of each day obligations, and study new talents to be able to be in demand in the present-day generation of automation (Misztal et. al. 2015, Abdollahi \& Babak 2019).

Many humans do not forget automation on a large scale; traditionally, that would be in environments including the huge three (Ford, Chrysler, and GM). But automation may be introduced to gadget gear in masses of other, more affordable tactics as well. A keep can begin with primary automation together with including a bar feeder to a lathe and construct from there, probably all the way to an entire, computerized cellular that handles the element from begin to finish. Automation can include robots that load and promote off machines or gantry structures which can be set up to the gadget device or as a stand-alone. Conveyors can circulate cloth from one place to the subsequent with turn or turn stations that rotate an element for the following operation. The bottom line is that by way of automating a production facility, a store can growth manufacturing, lessen cycle instances, improve satisfactory, lessen manufacturing lead instances, create a more comfortable place of work, and become extra aggressive.

But sure, demanding situations ought to be considered whilst implementing automation in the store. Some of those issues include ground area, rate, deciding what to automate, locating professional employees, and employee resistance.

The importance of recognition of human function in automated systems shows that regardless how advanced the system may be, it is important to be aware of all its factors and allocate tasks in relevance to it. The outcomes of the investigations confirmed that human beings cannot be eliminated from the procedures in automated structures. Introducing such systems into organization calls for that the manage ought to forecast the want of their control, usage and renovation, which have to be carried out with the engagement of employees.

\section{COMPONENTS OF AN INDUSTRIAL AUTOMATION SYSTEM}

The word reference characterizes automation as " The manner of creating an equipment, a process, or a system work robotically. "Automation Federation characterizes computerization as "the introduction and usage by age to display and address the introduction and conveyance of administrations and items". 
Automation Means usage of available advances to decrease the want of human labour. Industrial automation basically diminishes the need for human tangibly.

In Nineteen Sixties with the appearance of physical science, the logic gates started substitution the relays and auxiliary contactors within the management circuits. The hardware timers \& counters were replaced by electronic timers with blessings on energy saving, less maintenance \& bigger dependability, changes up to speed logic uphill, additional project time. In Seventies with the approaching of microprocessors and associated peripheral chips, the total method of technology underwent a radical improvement towards better automation system. The programmable controllers have in recent years provides unprecedented growth in Industrial Automation. It is effectively utilized in applications starting from straightforward management like the substitution tiny variety of relays to complicated automation issues. The advantages are: power saving, easy upkeep, in your price range, better reliability, terrific flexibility, shorter venture time, easier garage, archiving and documentation. Sensors with transmitter are placed within the field that experience the parameter and send the analog signal to the management hardware.

Industrial Automation additionally includes a high-quality, quantity of hardware technology, related to instrumentation and sensing, actuation and drives, electronics for sign conditioning, conversation, and display, embedded in addition to face-on self very own computing systems and lots of others.

As Industrial Automation frameworks increase greater refined concerning the capacity what is greater, calculations they use, as they incorporate massive locales of hobby that includes various devices or the whole of an assembling unit, or even some of them, and as they incorporate creation with numerous zones of business, which incorporates, pay and client care, fund and the entire flexibly chain of the enterprise, using IT increments significantly. In any case, the abatement level automation systems that only adapt to character or, at the top, a lot of machines, utilize IT and a mere quantity of system, gadgets and hooked up processing.

The financial association of extension emerges in various divisions of handing over, besides possibly the maximum excessive dominatingly in digital object fabricating in which complete item presence cycle, from hypothesis to commercial middle, is acted right away, if not weeks. In this manner, to draw back an possibility to market it remarkably utilization of the programmed equipment is commanded in all levels of the object lifestyles cycle. Moreover, on the grounds that a vast type of product ought to be engineered inside the manner of life, length of a production plant, brief programmability and reconfigurability of machines and strategies end up a key necessity for business accomplishment. Such a mechanized creation framework moreover permits the commercial enterprise to make the maximum a much bigger marketplace and moreover secures itself in opposition to variances popular for a given gloriousness of product. To be sure, it is far being pushed with the manual of the economic system of diploma, and empowered by means of technique for Industrial Automation Technology that Flexible Manufacturing (i.e., creating numerous objects with the indistinguishable gadget) has been imagined expanding the quantity of turning in.

Industrial automation is that the use of administration devices like PC/PLCs/PACs etc. to regulate industrial processes and machinery by removing the maximum amount labor intervention as potent, and substitution risky assembly operations with automatic ones. Industrial automation is closely coupled to regulate engineering. Three categories of automations (Figure 1) are Fixed Automation (Hard automation), Programmable Automation, Flexible Automation (Soft automation), Integrated Automation.

Computerization frameworks are probably ordered depending on the adaptability and segment of reconciliation in creation framework tasks. Different mechanization structures may be categorized as follows:

- Fixed Automation: It is used in high quantity generating with a committed device, which has a hard and fast association of responsibilities and supposed to be powerful for this set. Persistent 


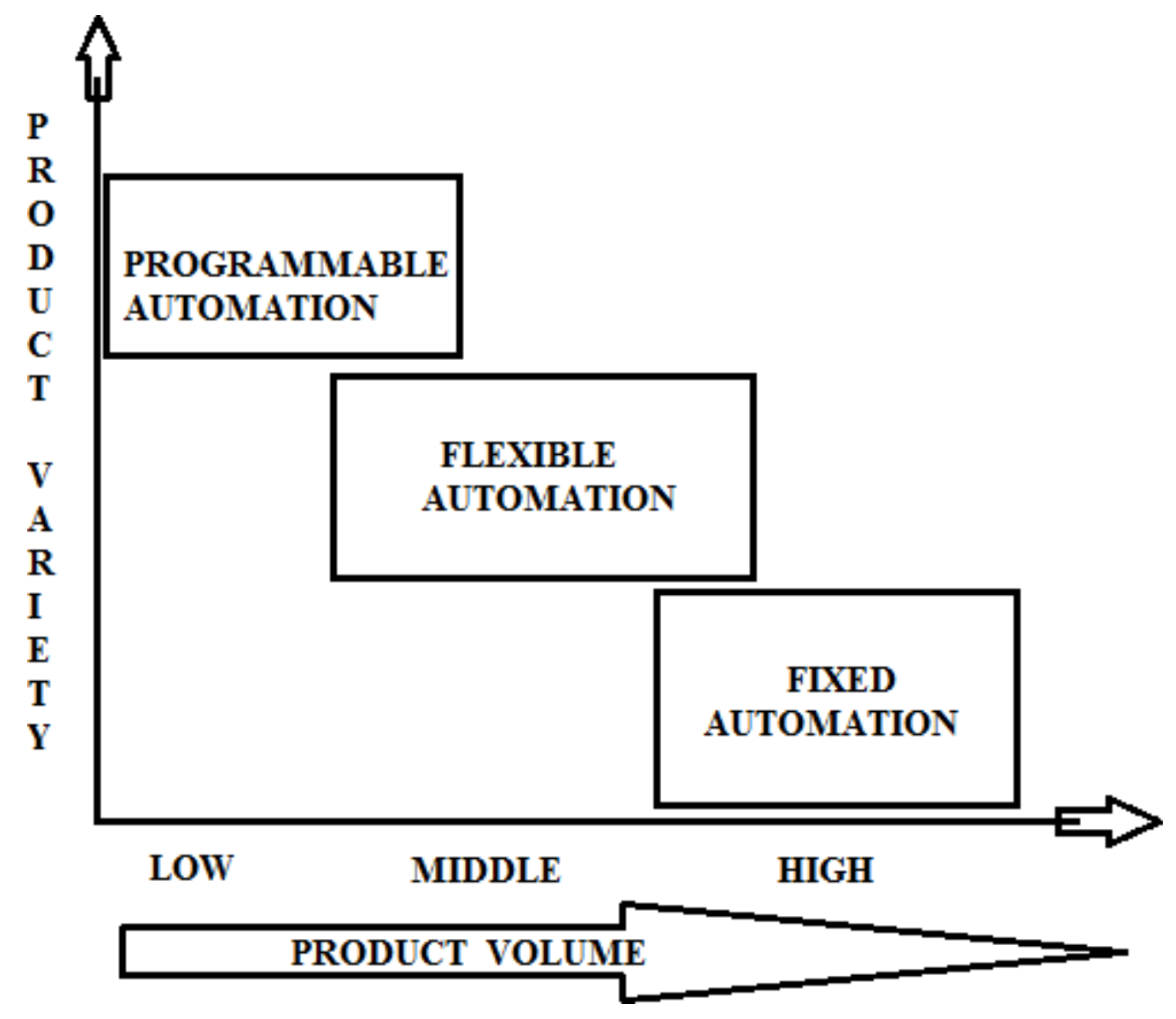

float and Discrete Mass Production structures make use of this robotization. In instance Refining Process, Transports, Paint Shops, Transfer traces, etc. A technique the utilization of computerized equipment to carry out fixed and dreary duties on request to offer a high quantity of comparative additives.

- Programmable Automation: It is utilized for a varied collection of activity and setup of the machines the usage of electronic controls. Be that as it can, non-insignificant programming enterprise might be needed to reinvent the machine or association of activities. Interest inside the programmable contraption is considerably less on account that the assembling manner is not changed typically. It is commonly applied in the Batch method wherein the technique cross is low and object quantity is medium to unreasonable, and now and again in mass assembling too. For example, In Steel Rolling Plants, Paper Mills, and several others also.

- Adaptable Automation: It is utilized in Flexible Manufacturing Systems (FMS) that is continually PC managed. Human administrators gracefully high-stage guidelines inside the shape of codes went into PC spotting objects and its district inside the arrangement and the lessening degree adjustments are cultivated robotically. Every introduction device receives settings/orders from the computer. These mechanized burdens/empty required devices additionally, complete their making ready orders. After preparing, stock is robotically moved to the subsequent system. It is usually utilized in technique shops and bunch strategies wherein object assortments are high and 
procedure volumes are medium to low. Such structures in the main make use of Multipurpose CNC machines, Automated Guided Vehicles (AGV), and so forth.

Form of automation recommends for production:

1. Light bulbs - Fixed automation

2. Garments - Flexible automation

3. Textile - Programmable automation

4. Cement - Programmable automation

5. Printing - Flexible automation

6. Pharmaceuticals - Flexible automation

7. Toys - Flexible automation

The mechanized variables of the creation machine are probably remoted into lessons: Automation of the assembling structures within the assembling unit, computerization of the assembling help structures in current assembling systems and the second one is the classifications cowl to a degree, since the programmed creation system handling the manufacturing plant ground is frequently done through strategies for PC systems and recognized with the electronic design support mechanism and the executive's facts device running the plant and superior quantity.

In a business setting, we will characterize computerization as an innovation this is associated with the utilization of mechanical, electrical/electronic, and PC principally based frameworks administer the assembling framework.

Case incorporates:

1. The transfer follows that play out a progression of machining activity

2. Mechanical get-together machines

3. Logistic help equipment

4. Numerically controlled machine gear

5. Remarks control systems

6. Computerized assessment framework for remarkable oversee

7. Automated material overseeing framework and capacity gadget to consolidate producing activity

8. CAD/CAM devices and robots are mechatronic contraptions that help business mechanization

Automation of the manufacturing aid structures is aimed at reducing the quantity of guide and clerical effort in product design, production planning and control and the agency features of the business enterprise. Computer the era is used to enforce automation of the producing systems in the manufacturing facility.

Automation Tools: ANN, DCS, HMI or TOUCH SCREEN (TS), PLC, SCADA, Variable frequency drive (VFD), Instrumentation, Motion control, Robotics, etc.

\subsection{Benefits of Industrial Automation and its Application}

Generally, the automation is the allocation of human control function over the technical equipment. The reasons behind the industrial automation are:

1. Improve safety

2. Less paperwork

3. Identify unused resources. 
4. Easily manage decentralized teams in different time zones.

5. Reduced routine manual tasks

6. Better process visibility

7. Improved control strategies

8. To reduce routine manual and clerical tasks

9. To mitigate the effects of labor shortages

10. Shorter workweeks for labour

11. Reduced factory lead times

12. More efficient use of materials

13. Reduced labor cost or production cost

14. Improved product quality

15. Assisted remote monitoring

16. Accomplish processes that cannot be done manually

17. Allow new business opportunities

Various application field of automation:

1. Swarms of microscopic robots for medical diagnosis

2. Pharmaceutical automation

3. Automated medication dispensing devices

4. Automation technologies in operation room

5. Automation in manufacturing industry

6. Automation in defence

7. Automation in mining

8. Automated drilling

9. Automated exploration

10. Automated trucks

11. Automation in agriculture

12. Switching in telephone networks

13. Stabilization of ships and aircraft

14. Automation in signaling and communication

15. Digitally controlled car engines

16. Biometric access control

17. RFID access control, etc.

Open-source hardware design may be a mechanical drawing, schematics, B.O.M, HDL source code and integrated circuit layout. In addition to the software that drives the hardware are all released with the OSH approach.

\subsection{Challenges of Automation}

The time and openings are now directly here. Advanced assembling can convey new chances, new markets, and new degrees of proficiency and worth decrease never thought doable. However, one initially must be eager to receive a new way of reasoning that virtually assembling requires. This computerized change results from what business analysts who study logical advancement and specialized change calls a universally useful innovation - that is, one that has the ability to consistently change itself, dynamically spreading out, and boosting profitability over all areas and ventures.

Notwithstanding rebuilding employments and capacities, it's likewise updating businesses, for example, retailing and distributing and conceivably - inside the imminent future - trucking and banking. 
Numerous innovation is joining these days to characterize "prevalent" production. The blending of the physical what's more, virtual has caused a global in which individuals and PC frameworks talk and team up. The expansion of cloud and robotization advances will bring about progressively consistent and continuous reproduction, work process, and coordinated effort to change the design of the new products and lift up the improved method. Items and devices are no longer offered on a unique case establishment. Presently, empowered with sensors and correspondences usefulness, makers have the opportunities for longer-term and routine bearer income streams. Assembling is advancing to the factor where mechanical enhancements and upgrades will originate from machines and frameworks themselves, through prescient examination, predominant controls, and more intelligent frameworks. Dynamic mechanization innovation with cutting edge programming program frameworks, mechanical technology, and complex new creation procedures. Investments in academics and the social safety together in the social settlement with industry provides the workplace profitable and protections.

If carefully controlled, changes in manufacturing and intake patterns ought to create environmental enhancements as compared with the procedures used today. But without right environmental goals and control structures, automation alone should have huge negative effects - particularly on energy use (and emissions generated from the current electricity mix), aid use and ecosystems. Now, it wouldn't be truthful if we didn't mention the downsides to automation. Fortunately, there are now not lots of them: Integration compatibility of the system: Industry needs to ensure that the platform it picks is compatible with the required existing systems. Is there an API supply to guarantee compatibility, or it will want to add programming sources to make sure that the management identifies the investment they will get before deciding to adopt the software for their system.

\subsubsection{Complexity}

Sometimes, the tasks the manufacturing industries have got to finish on each day organization can be complex. The software program can do it for you. Many carriers offer related offerings to establish the workflow inside the interface or make certain you can without difficulty configure the device in keeping with your business techniques.

\subsubsection{Security}

We regularly hear about troubles related to laptop protection. Indeed, ordinary there's infinite hacking attempts. Of direction, digitalization opens the door to this exposure, but the exceptional solutions usually improve their security features.

Automation in manufacturing (Figure 2) is the manner of the use of manufacturing management software and hardware or robotic application to perform a manufacturing facility when making a bodily product. These tools are constructed to perform operations to help corporations with responsibilities such as processing, meeting, inspection, inventory control, and production planning.

Automation is the software program of the automated gadget in a way or system. By the method of automation, manufacturing and exclusive business strategies are these days capable of the function at exceptional speeds way to the immoderate pace and accuracy of contemporary-day virtual gadgets. In current worldwide, machines take the biggest element within the method through the manner of converting most or all the positions wherein an ordinary takes vicinity, together with meeting or method control. This shift in methodology implies a decreased demand for the unskilled hand of labor to carry out and display the method, at the same time as generating a higher name for control structure experts who create and put in force automated approach of production. Such systems are, broadly speaking, composed of 3 number one organizations of components, which the professional has for you to configure and puzzle collectively properly into a layout that incorporates the hobby(s) required. These are the following. 


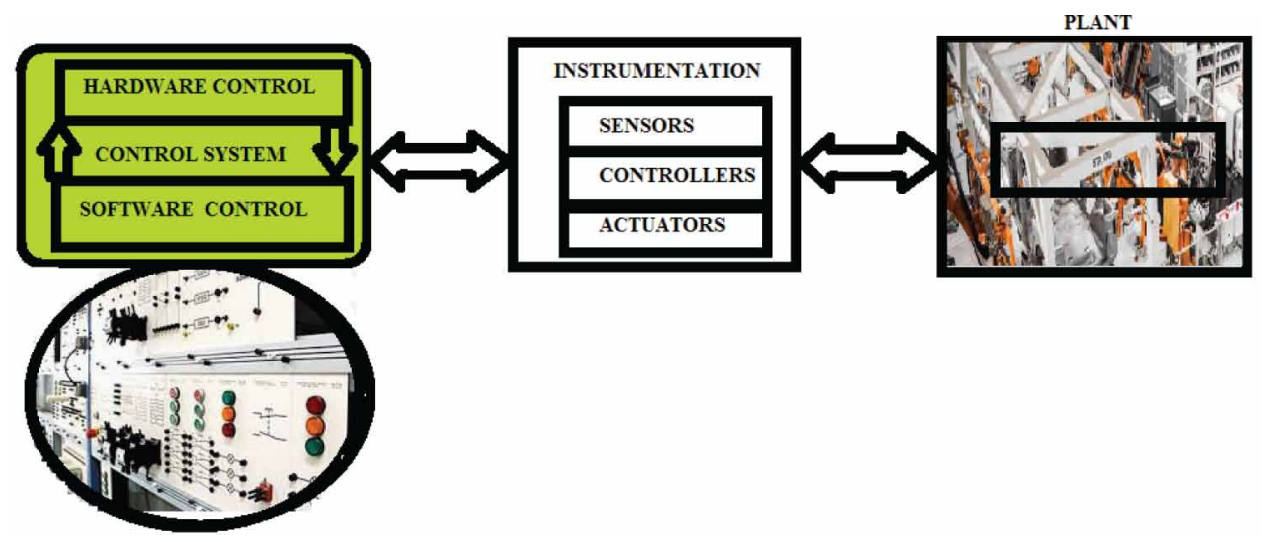

\subsubsection{Control Unit}

The "brains" of the machine, it receives the data from the sensors and controls the actuators in addition to hosting the UI. This device wants to be programmed to enforce the common feel required to complete the approach. Examples of such control structures are PLCs, microcontrollers, and any type of laptop which helps I/Os.

\subsubsection{Sensors}

These acquire statistics from the device and switch it to the control unit. In many instances, they need to be programmed for a selected purpose (inclusive of output signal type, operational range, and so forth.) and typically want to be chosen to be well suitable with the handling unit. Examples of inputs are restriction switches, strainmeters, and temperature meters.

\subsubsection{Actuators}

The gadgets which flow into or control a mechanism or machine, commonly powered through using an electrical supply. The actuators are the automated analogy of human fingers, bodily adjusting the variables to be managed. Common examples are solenoid and motorized valves, vehicles, and pneumatic jacks.

A technique is the sum of all connecting methodology inside a machine that changes and shops texture, power or data. A specialized technique is a methodology wherein its physical parameters are recorded and provoked by method for specialized methods.

\section{PROCESS MANAGEMENT AND AUTOMATION FUNCTIONS}

The phrase 'automation' is derived from historical Greek words of Auto (method 'self') Matos (manner 'moving'). Automated production systems function in the manufacturing unit of the physical product. They perform operations along with processing, meeting, inspection, or material coping with, in some cases conducting greater than one of these operations inside the equal gadget. Sequential manipulates may be either in a set series or to a logical one to be able to perform distinctive actions relying on various machine states. States check with the various conditions which could occur in a use or sequence state of affairs of the gadget. An instance is an elevator, which uses good judgment based totally in the device state to perform sure movements in response to its country and operator input. For instance, if the operator presses the ground on, the system will respond relying on whether or not the elevator is stopped or shifting, going up or down, or if the door is open or closed. 
Automation is the conversion of a part of a set method, a system, or device to computerized in the region of human operation or manage. Automation does now not in fact switch human abilities to machines, but consists of a deep reorganization of the artwork process, in the path of which every the human and the device functions are redefined. Early automation relied on mechanical and electromechanical manipulate gadgets; over the past 40 years, however, the PC frequently became the foremost vehicle of automation. Modern automation is commonly associated with computerization. Today's age will emphasize on the development, social and economic aspects of enterprise automation, focusing on the computerization of producing, engineering, and managerial obligations.

The modern computerization framework incorporates the handiest five key added substances: (an) activity component, (b) detecting or sensing instrument, (c) control component, (d) decision component, and (e) program. Activity factors of components are the major parts of automation and have a capacity to perform the assigned task.

Computers can carry out each sequential control and feedbacks manage, and commonly PC will do each in application. Programmable common sense controllers (PLCs) are a sort of unique purpose microprocessor that changed many hardware components including timers and drum sequencers utilized in relay common sense type systems. Motive process computer systems more and more changed stand-alone controllers, with a single pc able to perform the operations of masses of controllers. Process manage computers can process information from a community of PLCs, gadgets, and controllers to be able to put into effect ordinary (along with PID) control of man or woman variables or, in some instances, to implement complex control algorithms the use of more than one inputs and mathematical manipulations. They also can analyze facts and create actual-time graphical displays for operators and run reports for operators, engineers, and management.

As compared with guide structures, automation systems offer advanced performance in phrases of precision, strength, and pace of operation. In commercial automation manipulate, a huge variety of system variables consisting of temperature, drift, strain, distance, and liquid ranges can be sensed concurrently. All these variables are received, processed and managed by complicated microprocessor systems or PC based facts processing controllers. The control systems blended with monitoring tailored to the running surroundings within the industry permit for a flexible and green reliable production device.

In the computer technique manipulation, a digital PC is used to direct the operations of a manufacturing way. Although one of a kind automated systems are normally managed by way of the use of pc, the term pc technique management is generally related to continuous or semi-continuous production operations associated with substances such as chemical substances, petroleum, ingredients, and sure number one metals. In these operations, the goods are typically processed in gas, liquid, or powder form to facilitate the glide of the fabric through the diverse steps of the producing cycle. In addition, those merchandises are typically heavily produced. Because of the benefit of coping with the product and the massive volumes involved, an immoderate diploma of automation has been finished in those industries.

The current computer technique control device typically consists of the subsequent: (a) size of critical approach variables which includes temperature, go with the flow fee, and pressure, (b) execution of some optimizing method, (c) actuation of such gadgets as valves, switches, and furnaces that permit the manner to enforce the gold fashionable approach, and (d) technology of news to control indicating system repute, production overall performance, and product great.

As per Marco Annunziata, Chief Economist, GE, "Digital innovations of advanced manufacturing will increase the talents of workers at distinctive tiers of the skills distribution. Different and higher capabilities can be wished, but the era itself will assist within the procedure."

Automation can improve safety and remove risk of human error but at the same time some unskilled workers may be displaced. This creates winners and losers that fingers to possibility increase in inequality. As a result of this the automation may increase its monopoly power. But ultimately the automation adopted corporate will get higher labour productivity and higher wages or profit. 
Followings are some important areas inside the industry, where process automation can be easily adoptable (even if for small or medium scale industries) to increase operational efficiency and also improve customer relationship:

1. Research and development

2. Procurement

3. Sales and order processing

4. Distribution and logistics

5. Customer service

6. Compliance

7. Production

8. Operations

\section{EFFECT OF AUTOMATION ON SOCIETY AND ENVIRONMENT}

An automation venture will boom productiveness because of will increase in manufacturing and reductions in labour content material. Over the years, productivity gains have led to reduced charges for products and multiplied prosperity for society. The automation technologies in technical and socio-technical systems has gradually exacerbated their impact on human beings of their existence and on society in large. In turn, relationships among people and technologies are getting an increasing number of understandings intensive so one can better apprehend. One primary of communication about the approaching transformation has focused on the converting position of human in an age of automation. In fact, present technology allows automation of $1 / 2$ of all sports human beings are presently paid to do. They also substantially transform cutting-edge commercial enterprise fashions and manufacturer-client relationships and create new employment opportunities for the ones capable of use them.

Automation has the ability to transform future jobs and the structure of the labor pressure. While automation boosts economic growth, creates jobs, and improves living requirements, it could additionally gift serious challenges for employees and groups, including task displacement, disruptions to neighborhood economies, converting talent desires, and growing inequality. Labor studied, companies are considerably more likely to look mechanization developing their workforces instead of contracting them. "As corporations move advanced and towards digitalization," the record says, maximum will want extra human beings, no longer fewer. Automation is good news for processseekers, in the event that they have the talents. As world is entering to digitalization and adopting diverse automation system of their paintings subculture within the latest superior technology arena. Surely, many roles could be impacted whilst many jobs are created. Tentative percent on job threats because of industrial automation is shown in Figure 3.

The realities outline says that simultaneously as the general number of employments has quickened, the vast majority of those are in occupations which can be at low or medium threat. That demonstrates, it says, that the work commercial center might be changing over to occupations that require progressively mind boggling and significantly fewer standard abilities. Policymakers and business venture pioneers need to consider how they work together to address. Advanced technology is growing up in our surrounding very rapidly. The move from regular assembling to pc-empowered endeavor took just about a century. Be that as it may, the move from non-open processing to billions of smart phones, huge systems, and the IoT has taken basically different numerous years. Late appropriation and sharpness of computerized reasoning calculations - provoked by means of getting right of section to huge realities and better equipment - handling capacities - are changing over the essence of blue and office employments. The following period of innovative development is as of now in progress: prevalent neural systems that examine, adjust, and react to circumstances. 


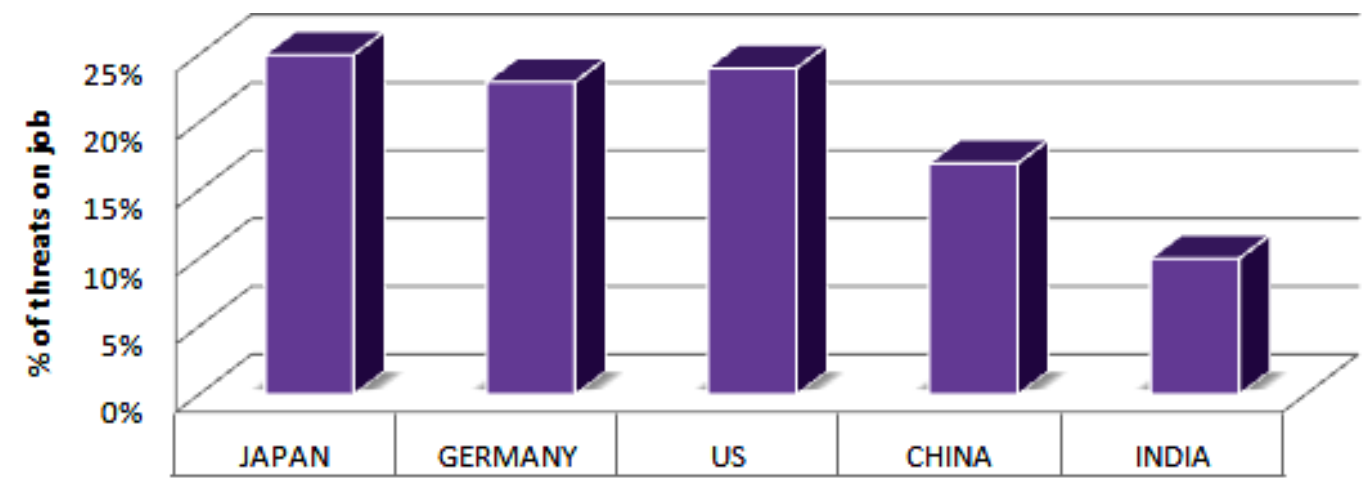

The truth of the issue is, even the popularity is probably going to require progressively consideration on instruction. Organizations - their biggest test is finding ensured people to take the occupations they have open. The predetermination of expanded robotization will direct workforce advancement that is focused on preparing individuals for the universe of unrivaled assembling." Robotization incorporates supplanting commitments as of now executed by method for individuals with time, that could incorporate pc projects, calculations, or even robots.

Robots have had nearness in assembling for a considerable length of time, drove with the guide of the vehicle business. The present scenario is expanding hurriedly as innovation improves and expenses of mechanical autonomy drop. Numerous employments in assembling (counting constructing agent, fabricator, mechanical engineer, and more noteworthy) include finishing tedious, unsurprising obligations. Machines are as of now taking on probably a few segments of these occupations, and that style will plausibly keep up. Each casing should keep up in their brain that robots are taking commitments yet not employments, i.e, to work the automated again professionals, engineers are required. For the most part, robots aren't self-insurance ability. When the automated is made by methods for gathering innovation then just it takes taking care of guidance of giving over the compositions. It is fundamental to follow computerization as it can affect the work market, economy, and society. The level of employment at a high possibility of computerization diminished scarcely somewhere in the range of 2013 and 2018, from $7.9 \%$ to $7.3 \%$, even as the level of occupations at low and medium risk of automation has risen. The particular thought processes in the lower in the extent of jobs at unnecessary danger of mechanization are indistinct, yet it's miles conceivable that computerization of certain occupations has as of now happened too.

Take an example like Amazon, it added extra than 50,000 new robots in 2017, a hundred percentage increases from the preceding year. Estimates endorse $20 \%$ of its workforce may already be made of robots. When thinking about the overall threat of automation, the three occupations with the lowest risk of automation are clinical practitioners, better training coaching professionals, and senior experts of instructional establishments. These occupations are all considered excessive professional. Figure 4 shows the possibility of chance to new job for skilled person in various automation adopted corporation sector.

The computerized upheaval must be general and improved as opposed to ignored and stifled. The historical backdrop of ahead of time general-reason innovations exhibits that despite brief timeframe period disengagements, rearranging the economy around progressive innovation produces huge longlasting period benefits. This does now not invalidate a job for open strategies. Despite what might be expected, it's far precisely at examples of fabulous innovative trade that reasonable rules are required. The plants made by means of the time of steam furthermore introduced governs on long stretches of work, adolescent work, and production line conditions. 


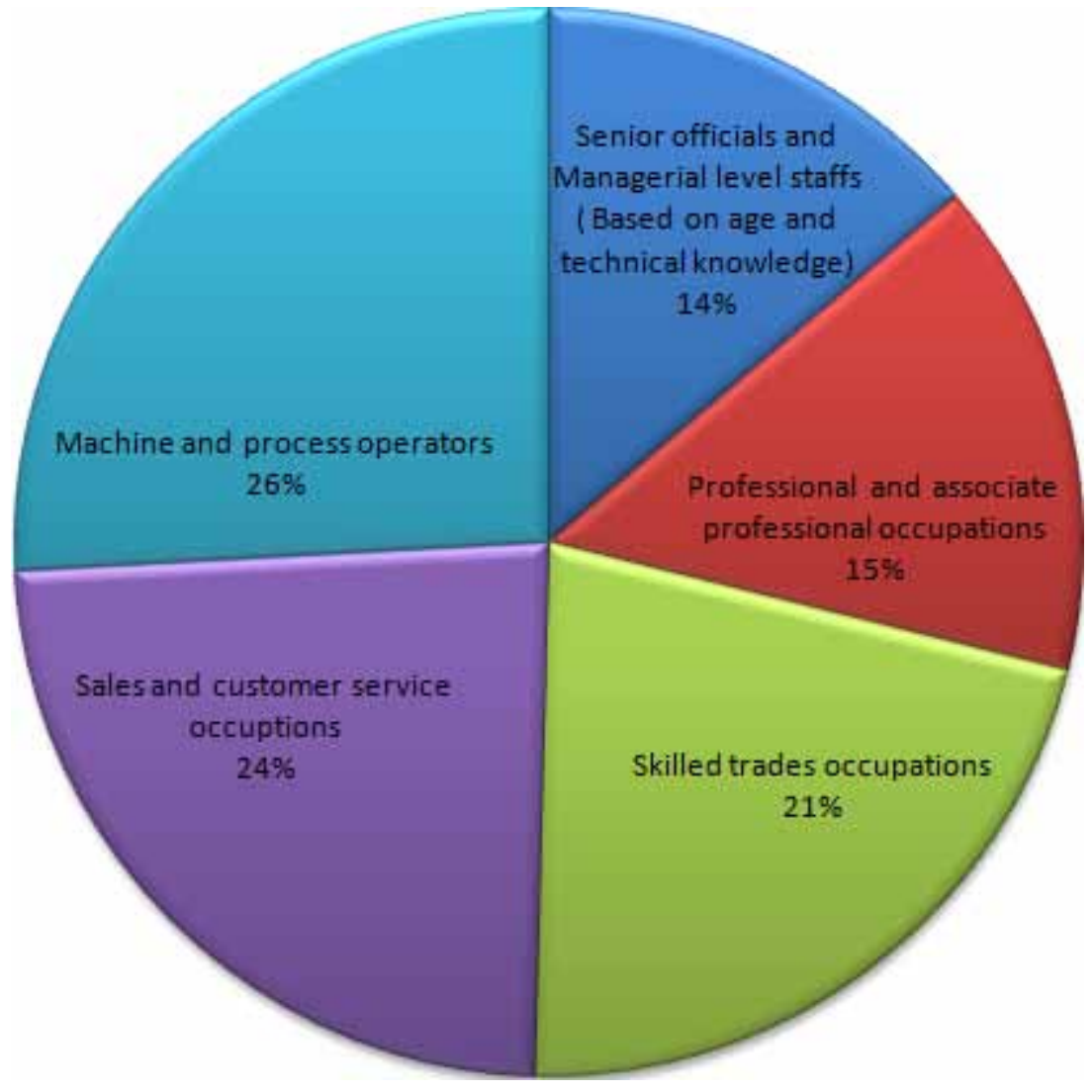

Training and rivalry approach can even be adjusted. Schools, colleges and universities should offer coming ages with the capabilities they have to work inside the rising economy. Be that as it may, social orders will likewise need to put a premium on retraining representatives whose capacities had been violated. So also, the redesign of creation puts new follows on challenge inclusion to verify that new procedures do now not become the area of certain organizations that start things out in a champ take-all lottery.

While the technical feasibility of automation is important, it isn't the high-quality detail as a way to steer the tempo and quantity of automation adoption. Other elements encompass the cost of growing and deploying automation answers for specific makes use of inside the place of business, the exertions-marketplace dynamics (including wonderful and quantity of hard work and associated wages), the blessings of automation past hard work substitution, and regulatory and social popularity.

The ability effect of automation on employment varies by means of the usage of career and zone. Activities maximum liable to automation consist of physical ones in predictable environments, including jogging gadgets and getting ready speedy food. Collecting and processing information are two distinctive classes of sports that increasingly more can be completed better and faster with machines. This has to displace huge quantities of hard work. It is important to observe that even if a few duties are automated, employment in some occupations won't decline but substitute workers may perform new obligations.

Jobs in unpredictable environments - occupations which consist of gardeners, plumbers, or vendors of an infant- and eldercare - may even commonly see much less automation in upcoming 
years also, due to the fact they're technically difficult to automate and frequently command pretty decrease wages, which makes automation a far less attractive commercial enterprise proposition.

\section{CONCLUSION}

Despite the social benefits that might result from retraining displaced employees for different jobs, in nearly all instances the worker whose task has been taken over through a machine undergoes a period of emotional pressure. In addition to displacement from paintings, the worker can be displaced geographically. In order to discover other paintings, a man or woman can also need to relocate, that's any other source of pressure. Automation is essentially utilized by machines instead of humans. There is a cycle of demand and supply. The automation got here into existence, there was want of required skilled humans after which there may be an improve in pleasant and amount, lower in manufacturing cost and a lower in selling rate. One most important circulation of communication about the coming near transformation has targeted on the changing role of human labour in an age of automation. Indeed, present technologies already permit automation of $1 / 2$ of all activities people are presently paid to do. They also significantly rework modern-day commercial enterprise models and producer-purchaser relationships and create new employment possibilities for the ones capable of use them. With an awesome chew of the worldwide labour pressure predicted to be looking for new jobs through 2030, a strong reaction is manifestly had to manipulate destructive social side outcomes.

With top principles and an ability to coordinate all through fringes, we will and should saddle these energizing innovation to upgrade prosperity without decreasing the power and excitement of the computerized age. 


\section{REFERENCES}

Abdollahi, H., \& Babak Ebrahimi, S. (2019). Modeling and Investigating the Economy and Production Structure of Iran Public Theater: A System Dynamics Approach. International Journal of System Dynamics Applications, 8(1), 60-78. doi:10.4018/IJSDA.2019010104

Abdollahi, H., \& Ebrahimi, S. B. (2019). Modeling and Investigating the Economy and Production Structure of Iran Public Theater: A System Dynamics Approach. International Journal of System Dynamics Applications, 8(1), 60-78. doi:10.4018/IJSDA.2019010104

Balfe, N., Sharples, S., \& Wilson, J. R. (2015). Impact of automation: Measurement of performance, workload and behaviour in a complex control environment. Applied Ergonomics, 47, 52-64. doi:10.1016/j.apergo.2014.08.002 PMID:25479974

Baporikar, N. (2020). Logistics Effectiveness Through Systems Thinking. International Journal of System Dynamics Applications, 9(2), 64-79. doi:10.4018/IJSDA.2020040104

Baumann, F., Friehe, T., \& Rasch, A. (2018). Product liability in markets for vertically differentiated products. American Law and Economics Review, 20(1), 46-81. doi:10.1093/aler/ahx013

Dutta, S., Sarkar, A., Samanta, K., Das, R., \& Ghosh, A. (2014). Supervision of control valve characteristics using PLC and creation of HMI by SCADA. Proc. 1st Int. Conf. Autom. Control Energy Syst. (ACES), 1-5. doi:10.1109/ACES.2014.6808025

Fallon, E. F., Chadwick, L., \& Putten, W. (2015). An analysis of the impact on trends in automation on human error potential in brachytherapy. Procedia Manufacturing, 3, 234-241. doi:10.1016/j.promfg.2015.07.134

Faunce A. W. (1965). Automation and the Division of Labor. University of California Press.

Forge, S., \& Blackman, C. (2010). helping hand for Europe: the competitive outlook for the EU robotics industry. EUR-Scientific and Technical Research Reports, Publications Office of the European Union.

Grossman, G. M., Helpman, E., Oberfield, E., \& Sampson, T. (2017). Balanced Growth Despite Uzawa. The American Economic Review, 107(4), 1293-1312. doi:10.1257/aer.20151739

Heck, B. S., Wills, L. M., \& Vachtsevanos, G. J. (2003). Software technologies for implementing reusable, distributed control systems. IEEE Control Syst. Mag.

Herrera, M. M., Carvajal-Prieto, L. A., Uriona-Maldonado, M., \& Ojeda, F. (2019). Modeling the Customer Value Generation in the Industry's Supply Chain. International Journal of System Dynamics Applications, 8(4), 1-13. doi:10.4018/IJSDA.2019100101

Kizito, A., \& Semwanga, A. R. (2020). Modeling the Complexity of Road Accidents Prevention: A System Dynamics Approach. International Journal of System Dynamics Applications, 9(2), 24-41. doi:10.4018/ IJSDA.2020040102

Lankisch, C., Prettner, A., \& Prskawetz, K. (2019). How can robots affect wage inequality? Economic Modelling, 81, 161-169. doi:10.1016/j.econmod.2018.12.015

Li, D., Guo, L. S., \& Zhang, C. (2013). Research on tension control with fuzzy PID control in the low relaxation PC strand wire based on mechanical automation. Advanced Materials Research, 738, 280-286. doi:10.4028/ www.scientific.net/AMR.738.280

Misztal, A., Butlewski, M., Jasiak, A., \& Janik, S. (2015). The human role in a progressive trend of foundry automation. Metalurgija, 54(2), 429-432.

Muir, B. M. (1987). Trust Between Humans and Machines, and the Design of decision aids. International Journal of Man-Machine Studies, 27(5-6), 527-539. doi:10.1016/S0020-7373(87)80013-5

Norman, D. (1978). The problem with automation: Inappropriate feedback and interaction, not over-automation. Proceedings of the Royal Society of London. Series B, Biological Sciences, 237, 585-593. PMID:1970904

Pan, Z., Polden, J., Larkin, N., Van Duin, S., \& Norrish, J. (2012). Recent progress on programming methods for industrial robots. Robotics and Computer-integrated Manufacturing, 28(2), 87-94. doi:10.1016/j.rcim.2011.08.004 
Parasuraman, R., \& Wickens, C. D. (2008). Humans: Still vital after all these years of automation. Human Factors, 50(3), 511-520. doi:10.1518/001872008X312198 PMID:18689061

Prettner, K. (2019). A note on the implications of automation for economic growth and the labor share. Macroeconomic Dynamics, 23(3), 1294-1301. doi:10.1017/S1365100517000098

Sheridan, T. B. (2011). Adaptive automation, level of automation, allocation authority, supervisory control, and adaptive control: Distinctions and modes of adaptation. IEEE Trans. Syst. Man Cybern. Syst. Hum., 41(4), 662-667. doi:10.1109/TSMCA.2010.2093888

Sjobakk, B., Thomassen, M. K., \& Alfnes, E. (2014). Implications of automation in engineer-to-order production: A case study. Adv. Manuf., 2(2), 141-149. doi:10.1007/s40436-014-0071-4

Skorupski, J., \& Uchronski, P. (2018). Evaluation of the effectiveness of an airport passenger and baggage security screening system. Journal of Air Transport Management, 66, 53-64. doi:10.1016/j.jairtraman.2017.10.006

Tarafdar, M., Beath, C. M., \& Ross, J. W. (2017). Enterprise cognitive computing applications - opportunities and challenges. IT Professional, 19(4), 21-27. doi:10.1109/MITP.2017.3051321

Vagia, M., Transeth, A. A., \& Fjerdingen, S. A. (2016). A literature review on the levels of automation during the years. What are the different taxonomies that have been proposed. Applied Ergonomics, 53, 190-202. doi:10.1016/j.apergo.2015.09.013 PMID:26467193

Zelinka, D., \& Amadei, B. (2019). Systems Approach for Modeling Interactions Among the Sustainable Development Goals Part 1: Cross-Impact Network Analysis. International Journal of System Dynamics Applications, 8(1), 23-40. doi:10.4018/IJSDA.2019010102

Kali Charan Rath $(P h D)$ is presently working as an Associate Professor in the Department of Mechanical Engineering, GIET University, Gunupur. He was awarded the prestigious Ph.D degree by NIT Jamshedpur in the year of 2013. Dr. Rath guided many M. Tech students for their projects and published the work in various journals. His interest research area: CAD/CAM, IoT, Robotics, Automation, Smart Manufacturing system, etc.

Surya Narayan Maharana is working as Asst. Professor in the Department of Mechanical Engineering, GIET University, Gunupur, Odisha. He guided B. Tech and M. Tech students for their project work.

Jyoti Rajak is working recently in IDTR, MSME Technology Centre, Jamshedpur. He has a wide experience in CNC and CAD/CAM area. His working area: CAD/CAM, Product modeling and simulation, CNC machining, automation, etc. 